\title{
FULLY BOUNDED NOETHERIAN RINGS AND FROBENIUS EXTENSIONS
}

\author{
S. CAENEPEEL AND T. GUÉDÉNON
}

\begin{abstract}
Let $i: A \rightarrow R$ be a ring morphism, and $\chi: R \rightarrow A$ a right $R$-linear map with $\chi(\chi(r) s)=\chi(r s)$ and $\chi\left(1_{R}\right)=1_{A}$. If $R$ is a Frobenius $A$-ring, then we can define a trace map $\operatorname{tr}: A \rightarrow A^{R}$. If there exists an element of trace 1 in $A$, then $A$ is right FBN if and only if $A^{R}$ is right FBN and $A$ is right noetherian. The result can be generalized to the case where $R$ is an $I$-Frobenius $A$-ring. We recover results of García and del Río and by Dăscălescu, Kelarev and Torrecillas on actions of group and Hopf algebras on FBN rings as special cases. We also obtain applications to extensions of Frobenius algebras, and to Frobenius corings with a grouplike element.
\end{abstract}

\section{INTRODUCTION}

A ring $A$ is called right bounded if every essential right ideal contains a non-zero two-sided ideal. $A$ is right fully bounded noetherian or right FBN if $A$ is noetherian, and $A / P$ is right bounded for every two-sided prime ideal $P$ of $A$. Obviously commutative noetherian rings are right FBN; more generally, noetherian PI-rings and artinian rings are FBN. A series of conjectures in classical ring theory can be proved in the case of rings with the FBN property, we refer to the introduction of [7] for a brief survey.

Assume that a finite group $G$ acts on $A$. García and Del Río [9] investigated the relationship between the FBN property for $A$ and its subring of invariants $A^{G}$. The main result is that, in case $A$ is right noetherian, the right FBN property for $A$ is equivalent to the right FBN property for $A^{G}$, if there exists an element in $A$ having trace 1. A similar statement was proved in [12] for rings graded by a finite group $G$. These results can be generalized to Hopf algebra actions (see $[7,10]$ ).

We have observed that the methods introduced in [9] can be applied in an apparently completely different situation. Let $S$ be a Frobenius algebra (with Frobenius system $\left.\left(e=e^{1} \otimes e^{2}, \bar{\nu}\right)\right)$ and $j: S \rightarrow A$ an algebra map, with $A$ a right noetherian ring. If there exists $a \in A$ such that $j\left(e^{1}\right) a j\left(e^{2}\right)=1$, then $A$ is right FBN if and only if $C_{S}(A)$ is right $\mathrm{FBN}$.

In this note, we propose a unified approach to these results, based on the concept of an $A$-ring with a grouplike character, as introduced in [6]. Basically, this consists of a ring morphism $i: A \rightarrow R$, together with a right $A$-linear map $\chi: R \rightarrow A$ such that the formula $a<r=\chi(a r)$ makes $A$ into a right $R$-module. The subring of invariants is defined as $B=\{b \in A \mid b \chi(r)=\chi(b r)\}$. The main result is basically

1991 Mathematics Subject Classification. 16W30.

Key words and phrases. Frobenius extension, Fully bounded noetherian ring, coring, Hopf algebra action, quasi-projective module.

Research supported by the project G.0278.01 "Construction and applications of noncommutative geometry: from algebra to physics" from FWO Vlaanderen. 
the following: if $R$ is a Frobenius $A$-ring, and $A$ is projective as a right $R$-module, then $A$ is right FBN if and only if $B$ is right FBN and $A$ is right noetherian. The methods of proof are essentially the same as in [9]. If $R$ is a Frobenius $A$-ring, then we can define a trace map $\operatorname{tr}: A \rightarrow B$, and $A$ is projective (and a fortiori quasi-projective) as a right $R$-module if and only if there exists an element of trace 1. The condition that $R$ is Frobenius can be relaxed in the sense that it suffices that $R$ is Frobenius of the second kind, with respect to a strict Morita context $(A, A, I, J, f, g)$. Then the trace map is a map $\operatorname{tr}: J \rightarrow B$.

The above mentioned results on group and Hopf algebra actions and extensions of Frobenius algebras can be obtained as special cases. We also present an application to Frobenius corings with a grouplike element.

\section{Rings With A GRouplike Character}

Let $A$ be an associative ring with unit. The category of $A$-bimodules ${ }_{A} \mathcal{M}_{A}$ is a monoidal category, and we can consider algebras in ${ }_{A} \mathcal{M}_{A}$. Such an algebra $R$ is a ring $R$ together with a ring morphism $i: A \rightarrow R$. The bimodule structure on $A$ is then given by $a r b=i(a) r i(b)$, for all $a, b \in A$ and $r \in R$. A right grouplike character on $R$ is a right $A$-linear map $\chi: R \rightarrow A$ such that

$$
\chi(\chi(r) s)=\chi(r s) \text { and } \chi\left(1_{R}\right)=1_{A},
$$

for all $r, s \in R$. We then say that $(R, i, \chi)$ is an $A$-ring with a right grouplike character. Right grouplike characters were introduced in [6]. The terminology is motivated by the fact that the dual of a coring with a grouplike element is a ring with a grouplike character (see Section 7). For all $a \in A$, we have that

$$
\chi(i(a))=\chi\left(1_{R} \cdot a\right)=\chi\left(1_{R}\right) a=1_{A} a=a,
$$

so $\chi \circ i=\operatorname{Id}_{A}$, and $i$ is injective, $\chi$ is surjective. Sometimes we will regard $i$ as an inclusion. $A$ is a right $R$-module, with right $R$-action

$$
a\llcorner r=\chi(a r) \text {. }
$$

$A$ is a cyclic right $R$-module, since

$$
a=\chi(i(a))=\chi\left(1_{A} i(a)\right)=1_{A} \rightarrow i(a),
$$

for all $a \in A$. For $M \in \mathcal{M}_{R}$, the submodule of invariants is defined as

$$
M^{R}=\{m \in M \mid m r=m \chi(r), \text { for all } r \in R\} .
$$

Let

$$
B=A^{R}=\{b \in A \mid b \chi(r)=\chi(b r), \text { for all } r \in R\} .
$$

Then $B$ is a subring of $A, M^{R}$ is a right $B$-module, and we have the invariants functor $(-)^{R}: \mathcal{M}_{R} \rightarrow \mathcal{M}_{B}$. We will now present some elementary properties of

$$
Q=R^{R}=\{q \in R \mid q r=q \chi(r), \text { for all } r \in R\} .
$$

Lemma 1.1. Let $(R, i, \chi)$ be an A-ring with a right grouplike character.

(1) $Q$ is a $(R, B)$-subbimodule of $R$;

(2) $\chi$ restricts to a $B$-bimodule map $\chi: Q \rightarrow B$;

(3) if $1_{R} \in Q$, then $i$ is an isomorphism of rings, with inverse $\chi$. 
Proof. 1) We refer to [6, Prop. 2.2].

2) For all $q \in Q$ and $r \in R$, we have

$$
\chi(q) \chi(r)=\chi(q \chi(r))=\chi(q r)=\chi(\chi(q) r),
$$

hence $\chi(q) \in B . \chi$ is right $A$-linear, so its restriction to $Q$ is right $B$-linear. For all $q \in Q \subset R$ and $b \in B$, we have, by the definition of $A^{R}=B$ that $b \chi(q)=\chi(b q)$, so $\chi$ is also left $B$-linear.

3) If $1_{R} \in Q$, then we have for all $r \in R$ that

$$
r=1_{R} r=1_{R} \chi(r)=1_{R} i(\chi(r))=i(\chi(r)) .
$$

It follows that $i$ is a left inverse of $\chi$. We have seen above that $i$ is always a right inverse of $\chi$, so it follows that $i$ is an isomorphism.

If $M \in \mathcal{M}_{R}$, then $\operatorname{Hom}_{R}(A, M) \in \mathcal{M}_{B}$, with right $B$-action $(f b)(a)=f(b a)$, for all $b \in B, f \in \operatorname{Hom}_{R}(A, M)$ and $a \in A$.

$\operatorname{End}_{R}(A)$ is a $B$-bimodule, with left $B$-action $(b f)(a)=b f(a)$, for all $b \in B$, $f \in \operatorname{End}_{R}(A)$ and $a \in A$.

Lemma 1.2. Let $(R, i, \chi)$ be an A-ring with a right grouplike character, and $M a$ right $R$-module.

(1) $\operatorname{Hom}_{R}(A, M) \cong M^{R}$ as right $B$-modules;

(2) $\operatorname{End}_{R}(A) \cong B$ as $B$-bimodules and as rings.

Proof. 1) For $f \in \operatorname{Hom}_{R}(A, M)$ and $r \in R$, we have

$$
f\left(1_{A}\right) r=f\left(1_{A}\llcorner r)=f(\chi(r))=f\left(1_{A}\right) \chi(r),\right.
$$

so $f\left(1_{A}\right) \in M^{R}$, and we have a well-defined map

$$
\phi: \operatorname{Hom}_{R}(A, M) \rightarrow M^{R}, \phi(f)=f\left(1_{A}\right) .
$$

$\phi$ is right $B$-linear since

$$
\phi(f b)=(f b)\left(1_{A}\right)=f\left(b 1_{A}\right)=f\left(1_{A} b\right)=f\left(1_{A}\right) b=\phi(f) b .
$$

The inverse of $\phi$ is given by the formula

$$
\phi^{-1}(m)(a)=m a,
$$

for all $m \in M^{R}$ and $a \in A$.

2) If $M=A$, then $\phi$ is also left $B$-linear since

$$
\phi(b f)=(b f)\left(1_{A}\right)=b f\left(1_{A}\right)=\phi(f) b .
$$

\section{Quasi-PRojective modules}

A right $R$-module $M$ is called quasi-projective if the canonical map $\operatorname{Hom}_{R}(M, M) \rightarrow$ $\operatorname{Hom}_{R}(M, M / N)$ is surjective, for every $R$-submodule $N$ of $M$. This means that every right $R$-linear map $f: M \rightarrow M / N$ factorizes through the canonical projection $p: M \rightarrow M / N$, that is, there exists a right $R$-linear map $g: M \rightarrow M$ such that $f=p \circ g$.

Proposition 2.1. Let $(R, i, \chi)$ be an A-ring with a right grouplike character. The following assertions are equivalent.

(1) A is quasi-projective as a right $R$-module; 
(2) for every right $R$-submodule $I$ of $A$, and every $a+I \in(A / I)^{R}$, there exists $b \in B$ such that $b-a \in I$;

(3) for every right $R$-submodule $I$ of $A,(A / I)^{R} \cong(B+I) / I$.

Proof. 1) $\Rightarrow 2$ ). Observe that

$$
(A / I)^{R}=\{a+I \in A / I \mid a \chi(r)-\chi(a r) \in I, \text { for all } r \in R\} .
$$

For $a+I \in(A / I)^{R}$, we have a well-defined right $A$-linear map

$$
f: A \rightarrow A / I, f\left(a^{\prime}\right)=a a^{\prime}+I .
$$

$f$ is right $A$-linear since

$$
\begin{aligned}
f\left(a^{\prime}\llcorner r)\right. & =a\left(a^{\prime}\llcorner r)+I=a \chi\left(a^{\prime} r\right)+I\right. \\
& =\chi\left(a a^{\prime} r\right)+I=\left(\left(a a^{\prime}\right)\llcorner r)+I=f\left(a^{\prime}\right)\llcorner r .\right.
\end{aligned}
$$

Let $p: A \rightarrow A / I$ be the canonical projection. Since $A$ is quasi-projective, there exists $g \in \operatorname{Hom}_{R}(A, A)$ such that $p \circ g=f$, that is $a a^{\prime}+I=g\left(a^{\prime}\right)+I$ and, in particular, $a+I=g\left(1_{A}\right)+I$, or $g\left(1_{A}\right)-a \in I$. Let us show that $b=g\left(1_{A}\right) \in B$. Indeed, for all $r \in R$, we have

$$
\begin{gathered}
\chi(b r)-b \chi(r)=\chi\left(g\left(1_{A}\right) r\right)-g\left(1_{A}\right) \chi(r)=\left(g\left(1_{A}\right)\llcorner r)-\left(g\left(1_{A}\right)\llcorner(i \circ \chi)(r))\right.\right. \\
=g\left(1_{A} \leftarrow r\right)-g((\chi \circ i \circ \chi)(r))=g(\chi(r))-g(\chi(r))=0 .
\end{gathered}
$$

$2) \Rightarrow 3)$. The map $B \rightarrow(A / I)^{R}, b \mapsto b+I$ induces a monomorphism $(B+I) / I \rightarrow$ $\overline{(A / I)^{R}}$. Condition 2) means precisely that this map is surjective.

$3) \Rightarrow 1$ ). Take a right $R$-linear map $f: A \rightarrow A / I$, with $I$ a right $R$-submodule of A. Then

$$
\chi\left(f\left(1_{A}\right) r\right)=f\left(1_{A}\right)\left\llcorner r=f\left(1_{A}\llcorner r)=f\left(\chi\left(1_{A} r\right)\right)=f(\chi(r))=f\left(1_{A}\right) \chi(r),\right.\right.
$$

so $f\left(1_{A}\right) \in(A / I)^{R} \cong(B+I) / I$. Take $b \in B$ such that $f\left(1_{A}\right)=b+I$, and consider the map $g: A \rightarrow A, g(a)=b a . g$ is right $R$-linear since

$$
g(a\llcorner r)=b(a\llcorner r)=b \chi(a r)=\chi(b a r)=(b a)\llcorner r=g(a) r .
$$

Finally

$$
(p \circ g)(a)=p(b a)=b a+I=f\left(1_{A}\right) a=f(a) .
$$

In Proposition 2.1, we characterize quasi-projectivity of $A$ as a right $R$-module. Projectivity has been characterized in [6, Prop. 2.4]:

Proposition 2.2. Let $(R, i, \chi)$ be an A-ring with a right grouplike character. The following assertions are equivalent.

(1) $A$ is projective as a right $R$-module;

(2) there exists $q \in Q$ such that $\chi(q)=1$.

We refer to [6, Prop. 2.4] for more equivalent properties.

Proposition 2.3. [1, 4.11] Let $R$ be a ring, $M$ a quasi-projective right $R$-module, and $N$ a noetherian right $R$-module. Then $\operatorname{Hom}_{R}(M, N)$ is a noetherian right $\operatorname{End}_{R}(M)$-module. 


\section{I-Frobenius RINGS}

Let $(R, i)$ be an $A$-ring, and $I=(A, A, I, J, f, g)$ a strict Morita context connecting $A$ with itself. We say that $R$ is an $I$-Frobenius $A$-ring if there exist an element $e=e^{1} \otimes u^{1} \otimes e^{2} \in R \otimes_{A} I \otimes_{A} R$ (summation understood implicitely) and an $A$ bimodule map $\bar{\nu}: R \otimes_{A} I \rightarrow A$ such that the following conditions are satisfied, for all $r \in R$ and $u \in I$ :

$$
\begin{aligned}
& r e^{1} \otimes u^{1} \otimes e^{2}=e^{1} \otimes u^{1} \otimes e^{2} r \\
& \bar{\nu}\left(e^{1} \otimes_{A} u^{1}\right) e^{2}=1_{R} ; \\
& e^{1} \otimes_{A} u^{1} \bar{\nu}\left(e^{2} \otimes_{A} u\right)=r 1_{R} \otimes_{A} u .
\end{aligned}
$$

If $I=\left(A, A, A, A, \mathrm{id}_{A}, \mathrm{id}_{A}\right)$, then the notion "I-Frobenius" coincides with the classical Frobenius property. Equivalent definitions are given in [4, Theorem 2.7]. $f: I \otimes_{A} J \rightarrow A$ and $g: J \otimes_{A} I \rightarrow A$ are $A$-bimodule isomorphisms, and

$$
f\left(u \otimes_{A} v\right) u^{\prime}=u g\left(v \otimes_{A} u^{\prime}\right) ; g\left(v \otimes_{A} u\right) v^{\prime}=v f\left(u \otimes_{A} v^{\prime}\right),
$$

for all $u, u^{\prime} \in I$ and $v, v^{\prime} \in J$. We will write

$$
f^{-1}\left(1_{A}\right)=\sum_{i} u_{i} \otimes v_{i} \in I \otimes_{A} J
$$

From the fact that $f$ is an $A$-bimodule isomorphism, it follows easily that

$$
\sum_{i} a u_{i} \otimes v_{i}=\sum_{i} u_{i} \otimes v_{i} a
$$

for all $a \in A$. We have the following generalization of [6, Theorem 2.7].

Theorem 3.1. Let $(R, i, \chi)$ be an I-Frobenius A-ring with a right grouplike character. Then $J$ is an $(R, B)$-bimodule, with left $R$-action

$$
r \cdot v=\sum_{i} \bar{\nu}\left(r g\left(v \otimes \chi\left(e^{1}\right) u^{1}\right) e^{2} \otimes_{A} u_{i}\right) v_{i}
$$

and we have an isomorphism $\alpha: J \rightarrow Q$ of $(R, B)$-bimodules.

Proof. The map $\alpha$ is defined by the formula

$$
\alpha(v)=g\left(v \otimes_{A} \chi\left(e^{1}\right) u^{1}\right) e^{2},
$$

for all $v \in J$. Let us first show that $\alpha(v) \in Q$. For all $r \in R$, we compute

$$
\begin{aligned}
& \alpha(v) r=g\left(v \otimes_{A} \chi\left(e^{1}\right) u^{1}\right) e^{2} r \stackrel{(4)}{=} g\left(v \otimes_{A} \chi\left(r e^{1}\right) u^{1}\right) e^{2} \\
& \stackrel{(1)}{=} g\left(v \otimes_{A} \chi\left(\chi(r) e^{1}\right) u^{1}\right) e^{2} \stackrel{(4)}{=} g\left(v \otimes_{A} \chi\left(e^{1}\right) u^{1}\right) e^{2} \chi(r)=\alpha(v) \chi(r) .
\end{aligned}
$$

$\alpha$ is right $B$-linear since

$$
\begin{aligned}
\alpha(v b)= & g\left(v b \otimes_{A} \chi\left(e^{1}\right) u^{1}\right) e^{2}=g\left(v \otimes_{A} b \chi\left(e^{1}\right) u^{1}\right) e^{2} \\
& =g\left(v \otimes_{A} \chi\left(b e^{1}\right) u^{1}\right) e^{2} g\left(v \otimes_{A} \chi\left(e^{1}\right) u^{1}\right) e^{2} b \stackrel{(4)}{=} \alpha(v) b,
\end{aligned}
$$

for all $b \in B$. The inverse $\beta$ of $\alpha$ is given by the composition

$$
Q \subset R \stackrel{R \otimes_{A} f^{-1}}{\longrightarrow} R \otimes_{A} I \otimes_{A} J \stackrel{\bar{\nu} \otimes_{A} J}{\longrightarrow} A \otimes_{A} J \cong J,
$$

or

$$
\beta(q)=\sum_{i} \bar{\nu}\left(q \otimes_{A} u_{i}\right) v_{i}
$$


for all $q \in Q$. Indeed, we compute for all $q \in Q$ that

$$
\begin{aligned}
\alpha(\beta(q)) & =g\left(\sum_{i} \bar{\nu}\left(q \otimes_{A} u_{i}\right) v_{i} \otimes_{A} \chi\left(e^{1}\right) u^{1}\right) e^{2} \\
= & \sum_{i} g\left(\bar{\nu}\left(q \otimes_{A} u_{i}\right) v_{i} \chi\left(e^{1}\right) \otimes_{A} u^{1}\right) e^{2} \stackrel{(8)}{=} \sum_{i} g\left(\bar{\nu}\left(q \otimes_{A} \chi\left(e^{1}\right) u_{i}\right) v_{i} \otimes_{A} u^{1}\right) e^{2} \\
= & \sum_{i} g\left(\bar{\nu}\left(q \chi\left(e^{1}\right) \otimes_{A} u_{i}\right) v_{i} \otimes_{A} u^{1}\right) e^{2} \stackrel{(4)}{=} \sum_{i} g\left(\bar{\nu}\left(\chi\left(e^{1}\right) \otimes_{A} u_{i}\right) v_{i} \otimes_{A} u^{1}\right) e^{2} q \\
= & \sum_{i} \bar{\nu}\left(\chi\left(e^{1}\right) \otimes_{A} u_{i}\right) g\left(v_{i} \otimes_{A} u^{1}\right) e^{2} q=\sum_{i} \bar{\nu}\left(\chi\left(e^{1}\right) \otimes_{A} u_{i} g\left(v_{i} \otimes_{A} u^{1}\right)\right) e^{2} q \\
& \stackrel{(7)}{=} \sum_{i} \bar{\nu}\left(\chi\left(e^{1}\right) \otimes_{A} f\left(u_{i} \otimes_{A} v_{i}\right) u^{1}\right) e^{2} q=\bar{\nu}\left(e^{1} \otimes_{A} e^{1}\right) e^{2} q=q .
\end{aligned}
$$

For all $v \in J$, we have that

$$
\begin{aligned}
\beta(\alpha(v)) & =\sum_{i} \bar{\nu}\left(g\left(v \otimes_{A} \chi\left(e^{1}\right) u^{1}\right) e^{2} \otimes_{A} u_{i}\right) v_{i}=\sum_{i} g\left(v \otimes_{A} \chi\left(e^{1}\right) u^{1}\right) \bar{\nu}\left(e^{2} \otimes_{A} u_{i}\right) v_{i} \\
= & \sum_{i} g\left(v \otimes_{A} \chi\left(e^{1}\right) u^{1} \bar{\nu}\left(e^{2} \otimes_{A} u_{i}\right)\right) v_{i} \stackrel{(6)}{=} \sum_{i} g\left(v \otimes_{A} \chi\left(1_{R}\right) u_{i}\right) v_{i} \\
= & \sum_{i} g\left(v \otimes_{A} u_{i}\right) v_{i} \stackrel{(7)}{=} \sum_{i} v f\left(u_{i} \otimes_{A} v_{i}\right)=v .
\end{aligned}
$$

This shows that $\alpha$ is an isomorphism of right $B$-modules. We can transport the left $B$-action on $Q$ to $J$ such that $\alpha$ becomes an $(R, B)$-bimodule map. This yields formula (9).

The composition

$$
\operatorname{tr}=\chi \circ \alpha: J \rightarrow Q \rightarrow B
$$

is a $B$-bimodule map (see Lemma 1.1), and will be called the trace map. It is given by the formula

$$
\operatorname{tr}(v)=\chi\left(g\left(v \otimes_{A} \chi\left(e^{1}\right) u^{1}\right) e^{2}\right) .
$$

Combining Proposition 2.2 and Theorem 3.1, we obtain the following result:

Proposition 3.2. Let $(R, i, \chi)$ be an I-Frobenius A-ring with a right grouplike character. The following assertions are equivalent.

(1) $A$ is projective as a right $R$-module;

(2) there exists $v \in J$ such that $\operatorname{tr}(v)=1_{B}$.

Now assume that $R$ is Frobenius $A$-ring, that is, $I=A$. Then the above formulas simplify. $e=e^{1} \otimes e^{2} \in R \otimes_{A} R, \bar{\nu}: R \rightarrow A$ is an $A$-bimodule map, and the trace map tr : $A \rightarrow B$ is given by

$$
\operatorname{tr}(a)=\chi\left(a \chi\left(e^{1}\right) e^{2}\right) .
$$

\section{FULLY BOUNDED NOETHERIAN RINGS}

We recall some definitions and basic results from [9]. Let $R$ be a ring, and $M, P \in$ $\mathcal{M}_{R}$. For a subset $X$ of $\operatorname{Hom}_{R}(P, M)$, we write

$$
r_{P}(X)=\cap\{\operatorname{Ker} f \mid f \in X\} .
$$

In particular, for $X \subset M \cong \operatorname{Hom}_{R}(R, M)$, we have

$$
r_{R}(X)=\{r \in R \mid x r=0\} .
$$


$M$ is called finitely $P$-generated if there exists an epimorphism of right $R$-modules $P^{n} \rightarrow M \rightarrow 0$.

$M$ is called $P$-faithful if $\operatorname{Hom}_{R}\left(P, M^{\prime}\right) \neq 0$, for every nonzero submodule $M^{\prime} \subset M$. $R$ is called right bounded if every essential right ideal contains a non-zero two-sided ideal. $R$ is called right fully bounded if $R / P$ is right bounded, for every two-sided prime ideal $P$ of $R$. A ring $R$ that is right fully bounded and right noetherian is called a right fully bounded noetherian ring or a FBN ring. Characterizations of right FBN rings are given in [9, Theorem 1.2]. For later use, we recall one of them.

Proposition 4.1. For a ring $R$, the following conditions are equivalent.

(1) $R$ is right $F B N$;

(2) for every finitely generated right $R$-module $M$, there exists a finite subset $F \subset M$ such that $r_{R}(M)=r_{R}(F)$.

A right $R$-module $P$ is called a right FBN-module if it is noetherian and for every finitely generated $P$-faithful right $R$-module $M$, there exists a finite subset $F \subset \operatorname{Hom}_{R}(P, M)$ such that $r_{P}(F)=r_{P}\left(\operatorname{Hom}_{R}(P, M)\right)$. We recall the following properties from [9].

Proposition 4.2. [9, Theorem 1.7] For a quasi-projective, noetherian right $R$ module $P$, the following assertions are equivalent:

(1) $\operatorname{End}_{R}(P)$ is right $F B N$;

(2) $P$ is an FBN right $R$-module.

Proposition 4.3. [9, Corollary 1.8] Let $P$ be a quasi-projective FBN right $R$ module, $Q$ a finitely $P$-generated right $R$-module, and $M$ a finitely generated $Q$ faithful right $R$-module. For every $X \subset \operatorname{Hom}_{R}(Q, M)$, there exists a finite subset $F \subset X$ such that $r_{Q}(X)=r_{Q}(F)$.

Proposition 4.4. [9, Corollary 1.9] A right noetherian ring $R$ is right FBN if and only if every finitely generated right $R$-module is FBN.

We can now state the main result of this paper.

Theorem 4.5. Let $(R, i, \chi)$ be an A-ring with a right grouplike character, and consider the following statements.

(1) $R \in \mathcal{M}_{A}$ is finitely generated and $A$ is right $F B N$;

(2) $R$ is right $F B N$ and $A$ is right noetherian;

(3) $B$ is right $F B N$ and $A$ is right noetherian.

Then 1) $\Rightarrow 2$ ).

If $A$ is quasi-projective as a right $R$-module, then 2$) \Rightarrow 3$ ).

If $A$ is projective as a right $R$-module and $R$ is an $I$-Frobenius $A$-ring for some strict Morita context $I=(A, A, I, J, f, g)$, then 3$) \Rightarrow 1)$ and the three conditions are equivalent.

Proof. 1) $\Rightarrow 2$ ). It follows from Proposition 4.4 that $R$ is an FBN right $R$-module. Let $M$ be a finitely generated right $R$-module; then $M$ is also finitely generated as a right $A$-module. We claim that $M$ is an $R$-faithful right $A$-module. Indeed, take a non-zero right $A$-module $M^{\prime} \subset M$. Since $M^{\prime} \cong \operatorname{Hom}_{A}\left(A, M^{\prime}\right)$, there exists a non-zero $f \in \operatorname{Hom}_{A}\left(A, M^{\prime}\right)$, and the composition $f \circ \chi: R \rightarrow M^{\prime}$ is non-zero, since $\chi$ is surjective.

Now take $P=R, Q=A$ in Proposition 4.3, and consider the subset $M \cong$ 
$\operatorname{Hom}_{R}(R, M) \subset \operatorname{Hom}_{A}(R, M)$. It follows that there exists a finite $F \subset M$ such that $r_{A}(F)=r_{A}(M)$. It then follows from Proposition 4.1 that $R$ is right FBN.

$2) \Rightarrow 3$ ). $A$ is a finitely generated (even cyclic) right $R$-module, so it follows from Proposition 4.4 that $A$ is an FBN right $R$-module. It then follows from Proposition 4.2 that $\operatorname{End}_{R}(A) \cong B$ is right $\mathrm{FBN}$.

$3) \Rightarrow 1$ ). We will apply Proposition 2.3 with $M=A$ and $N=R$. By assumption, $A$ is quasi-projective as a right $R$-module. Since $R / A$ is $I$-Frobenius, $R$ is finitely generated projective as a right $R$-module. Since $A$ is right noetherian, $R$ is also right noetherian.

It follows from Lemma 1.2, Proposition 2.3 and Theorem 3.1 that $\operatorname{Hom}_{R}(A, R) \cong$ $R^{R}=Q \cong J$ is noetherian as a right module over $\operatorname{End}_{R}(A) \cong A^{R}=B$. It then follows that $J$ is finitely generated as a right $B$-module. Let $\left\{e_{1}, \cdots, e_{k}\right\}$ be a set of generators of $J$ as a right $B$-module.

Recall that we have an $A$-bimodule isomorphism $f: I \otimes_{A} J \rightarrow A$. With notation as in Section 3, we have, for $a \in A$,

$$
f^{-1}(a)=\sum_{i=1}^{n} u_{i} \otimes_{A} v_{i} a \in I \otimes_{A} J .
$$

For every $i$, we can find $b_{i 1}, \cdots, b_{i k_{i}} \in B$ such that

$$
v_{i} a=\sum_{j=1}^{k_{i}} e_{j} b_{i j}
$$

We then easily compute that

$$
a=f\left(\sum_{i=1}^{n} u_{i} \otimes_{A} v_{i} a\right)=f\left(\sum_{i=1}^{n} \sum_{j=1}^{k_{i}} u_{i} \otimes_{A} e_{j} b_{i j}\right)=\sum_{i=1}^{n} \sum_{j=1}^{k_{i}} f\left(u_{i} \otimes_{A} e_{j}\right) b_{i j},
$$

and we conclude that $A$ is finitely generated as a right $B$-module.

Take $M \in \mathcal{M}_{A}$ finitely generated. Then $M$ is also finitely generated as a right $B$-module. We now show that $M$ is an $A$-faithful right $B$-module. Let $M^{\prime}$ be a non-zero right $B$-submodule of $M$, and take $0 \neq m^{\prime} \in M^{\prime}$. It follows from Proposition 3.2 that there exists $v \in J$ such that $\operatorname{tr}(v)=1_{B}$. The map $f: A \rightarrow M$, $f(a)=m^{\prime} \operatorname{tr}(v a)$ is right $B$-linear, and different from 0 since $f\left(1_{A}\right)=m^{\prime} \neq 0$.

Observe now that

- $B$ is a quasi-projective FBN right $B$-module;

- $A$ is a finitely $B$-generated right $B$-module;

- $M$ is a finitely generated $A$-faithful right $B$-module.

Applying Proposition 4.3 to $M \cong \operatorname{Hom}_{A}(A, M) \subset \operatorname{Hom}_{B}(A, M)$, we find that there exists a finite subset $F \subset M$ such that $r_{A}(F)=r_{A}(M)$. It then follows from Proposition 4.1 that $A$ is right FBN.

Remark 4.6. We do not know whether the implication 3$) \Rightarrow 1$ ) holds under the weaker assumption that $A \in \mathcal{M}_{R}$ is quasi-projective. The projectivity is used at the point where we applied Proposition 4.3. 


\section{Application to Frobenius algebras}

Let $k$ be a commutative ring, and consider two $k$-algebras $A$ and $S$, and an algebra map $j: S \rightarrow A$. All unadorned tensor products in this Section are over $k$. It is easy to establish that $\left(R=S^{\text {op }} \otimes A, i, \chi\right)$ with

$$
\begin{gathered}
i: A \rightarrow S^{\mathrm{op}} \otimes A, \quad i(a)=1_{S} \otimes a, \\
\chi: S^{\mathrm{op}} \otimes A \rightarrow A, \quad \chi(s \otimes a)=j(s) a
\end{gathered}
$$

is an $A$-ring with a right grouplike character. Also observe that the categories $\mathcal{M}_{R}$ and ${ }_{S} \mathcal{M}_{A}$ are isomorphic. For $M \in{ }_{S} \mathcal{M}_{A}$, we have that

$$
M^{R}=\{m \in M \mid s m=m j(s), \text { for all } s \in S\}=C_{S}(M) .
$$

In particular, $B=A^{R}=C_{S}(A)$ and

$$
Q=\left\{\sum_{i} s_{i} \otimes a_{i} \in S^{\mathrm{op}} \otimes A \mid \sum_{i} t s_{i} \otimes a_{i}=\sum_{i} s_{i} \otimes a_{i} j(t), \text { for all } t \in S\right\} .
$$

Consequently $A$ is projective as a right $R$-module if and only if there exists $\sum_{i} s_{i} \otimes$ $a_{i} \in Q$ such that $\sum_{i} j\left(s_{i}\right) a_{i}=1_{A}$.

From Proposition 2.1, it follows that $A$ is quasi-projective as a right $R$-module if and only if for every $(S, A)$-submodule $I$ of $A$ and $a \in A$ such that $a s-s a \in I$, for all $s \in S$, there exists $b \in B$ such that $a-b \in I$.

Assume that $S$ is a Frobenius $k$-algebra, with Frobenius system $\left(e=e^{1} \otimes e^{2}, \bar{\nu}\right)$. Then $S^{\text {op }}$ is also a Frobenius algebra, with Frobenius system $\left(e=e^{2} \otimes e^{1}, \bar{\nu}\right)$, and $S^{\text {op }} \otimes A$ is a Frobenius $A$-ring, with Frobenius system $(E, N)$, with $E=\left(e^{2} \otimes\right.$ $\left.1_{A}\right) \otimes_{A}\left(e^{1} \otimes 1_{A}\right)$ and

$$
N: S^{\mathrm{op}} \otimes A \rightarrow A, N(s \otimes a)=\bar{\nu}(s) a .
$$

We then have the isomorphism

$$
\alpha: A \rightarrow Q, \alpha(a)=e^{1} \otimes a j\left(e^{2}\right)
$$

and the trace map

$$
\operatorname{tr}: A \rightarrow B, \operatorname{tr}(a)=j\left(e^{1}\right) a j\left(e^{2}\right) .
$$

$A$ is projective as a right $R$-module if and only if there exists $a \in A$ such that $\operatorname{tr}(a)=1$.

Corollary 5.1. Let $S$ be a Frobenius algebra over a commutative ring $k$, and $j: S \rightarrow A$ an algebra map. Furthermore, assume that there exists a $\in A$ such that $\operatorname{tr}(a)=1$. Then the following assertions are equivalent:

(1) $A$ is right $F B N$;

(2) $S^{\mathrm{op}} \otimes A$ is right $F B N$ and $A$ is right noetherian;

(3) $B=C_{S}(A)$ is right $F B N$ and $A$ is right noetherian.

\section{Application to Hopf algeba actions}

Let $H$ be a finitely generated projective Hopf algebra over a commutative ring $k$, and $A$ a left $H$-module algebra. The smash product $R=A \# H$ is equal to $A \otimes H$ as a $k$-module, with multiplication given by the formula

$$
(a \# h)(b \# k)=a\left(h_{(1)} \cdot b\right) \# h_{(2)} k .
$$

The unit is $1_{A} \# 1_{H}$. Consider the maps

$$
i: A \rightarrow A \# H, i(a)=a \# 1_{H},
$$




$$
\chi: A \# H \rightarrow A, \chi(a \# h)=a \varepsilon(h) .
$$

Straightforward computations show that $(A \# H, i, \chi)$ is an $A$-ring with a left grouplike character. It is also easy to prove that

$$
A^{R}=\{a \in A \mid h \cdot a=\varepsilon(h) a, \text { for all } h \in H\}=A^{H}
$$

is the subalgebra of invariants of $R$.

In a similar way, we can associate an $A$-ring with right grouplike character to a right $H$-comodule algebra. We will discuss the left handed case here, in order to recover the results from $[7,9,10]$. The results from the previous Sections can easily be restated for rings with a left grouplike character.

Let $I=\int_{H^{*}}^{l}$ and $J=\int_{H}^{l}$ be the spaces of left integrals on and in $H . \quad I$ and $J$ are projective rank one $k$-modules, and $H / k$ is $I$-Frobenius (see for example [4, Theorem 3.4]). We need an explicit description of the Frobenius system. From the Fundamental Theorem, it follows that we have an isomorphism

$$
\phi: I \otimes H \rightarrow H^{*}, \phi(\varphi \otimes h)=h \cdot \varphi,
$$

with $\langle h \cdot \varphi, k\rangle=\langle\varphi, k h\rangle$. If $t \in J$, then

$$
\phi(\varphi \otimes t)(h)=\langle\varphi, h t\rangle=\langle\varphi, t\rangle \varepsilon(h),
$$

so $\phi$ restricts to a monomorphism $\tilde{\phi}: I \otimes J \rightarrow k \varepsilon$. If $I$ and $J$ are free of rank one, then $\tilde{\phi}$ is an isomorphism, as there exist $\varphi \in I$ and $t \in J$ such that $\langle\varphi, t\rangle=1$ (see for example [5, Theorem 31], [13]. Hence $\tilde{\phi}$ is an isomorphism after we localize at a prime ideal $p$ of $k$, and this implies that $\tilde{\phi}$ is itself an isomorphism. Consequently $J^{*} \cong I$. Consider $\tilde{\phi}^{-1}(\varepsilon)=\sum_{i} \varphi_{i} \otimes t_{i} \in I \otimes J$. Then

$$
\sum_{i}\left\langle\varphi_{i}, t_{i}\right\rangle=1 \text {. }
$$

Furthermore $\left\{\left(\varphi_{i}, t_{i}\right) \mid i=1, \cdots, n\right\}$ is a finite dual basis for $I$, so we have $t=$ $\sum_{i}\left\langle\varphi_{i}, t\right\rangle t_{i}, \varphi=\sum_{i}\left\langle\varphi, t_{i}\right\rangle \varphi$, for all $t \in J$ and $\varphi \in I$. $\phi$ induces an isomorphism

$$
\psi: H \rightarrow H^{*} \otimes J, \psi(h)=\sum_{i} h \cdot \varphi_{i} \otimes t_{i} .
$$

The inverse of $\psi$ is given by the formula

$$
\psi^{-1}\left(h^{*} \otimes t\right)=\left\langle h^{*}, \bar{S}\left(t_{(1)}\right)\right\rangle t_{(2)},
$$

where $\bar{S}$ is the inverse of the antipode $S$; recall from [13] that the antipode of a finitely generated projective Hopf algebra is always bijective. Indeed, it is straightforward to show that $\psi^{-1}$ is a right inverse of $\psi$. First observe that

$$
\psi\left(\psi^{-1}\left(h^{*} \otimes t\right)\right)=\sum_{i}\left\langle h^{*}, \bar{S}\left(t_{(1)}\right)\right\rangle t_{(2)} \cdot \varphi_{i} \otimes t_{i} .
$$

Now we compute for all $h \in H$ that

$$
\begin{gathered}
\left\langle h^{*}, \bar{S}\left(t_{(1)}\right)\right\rangle\left\langle t_{(2)} \cdot \varphi_{i}, h\right\rangle=\left\langle h^{*}, \bar{S}\left(t_{(1)}\right) \bar{S}\left(h_{(2)}\right) h_{(1)}\right\rangle\left\langle\varphi_{i}, h_{(3)} t_{(2)}\right\rangle \\
=\left\langle h^{*}, \bar{S}\left(h_{(2)} t_{(1)}\right) h_{(1)}\right\rangle\left\langle\varphi_{i}, h_{(3)} t_{(2)}\right\rangle \\
=\left\langle h^{*}, \bar{S}\left(1_{H}\right) h_{(1)}\right\rangle\left\langle\varphi_{i}, h_{(2)} t\right\rangle=\left\langle h^{*}, h\right\rangle\left\langle\varphi_{i}, t\right\rangle,
\end{gathered}
$$

where we used the fact that $\varphi_{i}$ and $t$ are integrals. It follows that

$$
\psi\left(\psi^{-1}\left(h^{*} \otimes t\right)\right)=\sum_{i} h^{*} \otimes\left\langle\varphi_{i}, t\right\rangle t_{i}=h^{*} \otimes t
$$


A right inverse of an invertible element is also a left inverse, so it follows that

$1_{H}=\psi\left(\psi^{-1}\left(1_{H}\right)\right)=\sum_{i}\left\langle\varphi_{i}, \bar{S}\left(t_{i(1)}\right)\right\rangle t_{i(2)}=\sum_{i}\left\langle\varphi_{i} \circ \bar{S}, t_{i(1)}\right\rangle t_{i(2)}=\sum_{i}\left\langle\varphi_{i} \circ \bar{S}, t_{i}\right\rangle 1_{H}$,

where we used the fact that $\varphi_{i} \circ \bar{S}$ is a right integral on $H$. We conclude that

$$
\sum_{i}\left\langle\varphi_{i}, \bar{S}\left(t_{i}\right)\right\rangle=1
$$

Consider the particular situation where $I$ and $J$ are free rank one modules. Then there exist free generators $\varphi_{1}$ of $I$ and $t_{1}$ of $J$ such that $\left\langle\varphi_{1}, t_{1}\right\rangle=1$. From (13) it follows that $\left\langle\varphi_{1}, \bar{S}\left(t_{1}\right)\right\rangle=1$. For arbitrary $\varphi=x \varphi_{1} \in I$ and $t=y t_{1} \in J$, it then follows that $\langle\varphi, t\rangle=x y\left\langle\varphi_{1}, t_{1}\right\rangle=x y=x y\left\langle\varphi_{1}, \bar{S}\left(t_{1}\right)\right\rangle=\langle\varphi, \bar{S}(t)\rangle$. Consider the case where $I$ and $J$ are not necessarily free, and take $\varphi \in I, t \in J$ and a prime ideal $p$ of $k$. Then the images of $\langle\varphi, t\rangle$ and $\langle\varphi, \bar{S}(t)\rangle$ in the localized ring $k_{p}$ are equal, since the integral space of the Hopf $k_{p}$-algebra $H_{p}$ is free. So we can conclude that

$$
\langle\varphi, t\rangle=\langle\varphi, \bar{S}(t)\rangle
$$

Lemma 6.1. Let $H$ be a finitely generated projective Hopf algebra over a commutative ring $k$. There exist $t_{i} \in J=\int_{H}^{l}$ and $\varphi_{i} \in I=\int_{H^{*}}^{l}$ such that $\sum_{i}\left\langle\varphi_{i}, t_{i}\right\rangle=1$. $H$ is an I-Frobenius $k$-algebra, with Frobenius system $(e, \bar{\nu})$ with

$$
\begin{aligned}
& e=\sum_{i} t_{i(2)} \otimes \varphi_{i} \otimes \bar{S}\left(t_{i(1)}\right) \\
& \bar{\nu}=\sum_{j} t_{j} \otimes \varphi_{j} \in(H \otimes I)^{*} \cong J \otimes H^{*}
\end{aligned}
$$

Proof. It is straightforward to show that $e \in C_{H}(H \otimes I \otimes H)$; this also follows from [4, Prop. 3.3], taking into account that $e=i^{\prime}(\varphi \otimes \bar{S}(t))$.

Write $e=e^{1} \otimes u^{1} \otimes e^{2} \in H \otimes I \otimes H$. We compute that

$$
\begin{aligned}
\bar{\nu}\left(e^{1} \otimes u^{1} \otimes e^{2}\right)=\sum_{i, j}\left\langle\varphi_{j}, t_{i(2)}\right\rangle\left\langle\varphi, t_{j}\right\rangle \bar{S}\left(t_{i(1)}\right) \\
=\sum_{i}\left\langle\varphi_{i}, t_{i(2)}\right\rangle \bar{S}\left(t_{i(1)}\right)=\sum_{i} \bar{S}\left(\left\langle\varphi_{i}, t_{i}\right\rangle 1_{H}\right) \stackrel{(12)}{=} 1_{H} .
\end{aligned}
$$

For all $\varphi \in I$, we calculate

$$
\begin{aligned}
e^{1} \otimes u^{1} & \bar{\nu}\left(e^{2} \otimes \varphi\right)=\sum_{i, j} t_{i(2)} \otimes \varphi_{i}\left\langle\varphi_{j}, \bar{S}\left(t_{i(1)}\right)\right\rangle\left\langle\varphi, t_{j}\right\rangle \\
\quad= & \sum_{i, j} 1_{H} \otimes \varphi_{i}\left\langle\varphi_{j}, \bar{S}\left(t_{i}\right)\right\rangle\left\langle\varphi, t_{j}\right\rangle=\sum_{i} 1_{H} \otimes \varphi_{i}\left\langle\varphi, \bar{S}\left(t_{i}\right)\right\rangle \\
\stackrel{(14)}{=} & \sum_{i} 1_{H} \otimes \varphi_{i}\left\langle\varphi, t_{i}\right\rangle=1_{H} \otimes \varphi .
\end{aligned}
$$

It now follows from [4, Theorem 3.1] that $(e, \bar{\nu})$ is a Frobenius system. 
Proposition 6.2. Let $H$ be a finitely generated projective Hopf algebra over a commutative ring $k$, and $A$ a left $H$-module algebra. Then $A \otimes H$ is an $A \otimes I$ Frobenius A-algebra, with Frobenius system $(E, N)$, with

$$
\begin{array}{r}
E=E^{1} \otimes_{A} U^{1} \otimes_{A} E^{2}=\left(1_{A} \# e^{1}\right) \otimes_{A}\left(1_{A} \otimes u^{1}\right) \otimes_{A}\left(1_{A} \# e^{1}\right) \\
=\sum_{i}\left(1_{A} \# t_{i(2)}\right) \otimes_{A}\left(1_{A} \otimes \varphi_{i}\right) \otimes_{A}\left(1_{A} \# \bar{S}\left(t_{i(1)}\right)\right), \\
N:(A \# H) \otimes_{A}(A \otimes I) \cong A \# H \otimes I \rightarrow A, \\
N(a \# h \otimes \varphi)=a \bar{\nu}(h \otimes \varphi)=\sum_{j} a\left\langle\varphi_{j}, h\right\rangle\left\langle\varphi, t_{j}\right\rangle .
\end{array}
$$

Here we used the notation introduced above.

Proof. The proof is an adaptation of the proof of [6, Proposition 5.1]. Let us first show that $E$ satisfies (4).

$$
\begin{aligned}
\sum_{i}\left(1_{A} \# t_{i(2)}\right) \otimes_{A}\left(1_{A} \otimes \varphi_{i}\right) \otimes_{A}\left(1_{A} \# \bar{S}\left(t_{i(1)}\right)(a \# h)\right. \\
=\sum_{i}\left(1_{A} \# t_{i(3)}\right) \otimes_{A}\left(1_{A} \otimes \varphi_{i}\right) \otimes_{A}\left(\bar{S}\left(t_{i(2)}\right) \cdot a \# \bar{S}\left(t_{i(1)}\right) h\right) \\
=\sum_{i}\left(1_{A} \# t_{i(3)}\right) \otimes_{A}\left(\bar{S}\left(t_{i(2)}\right) \cdot a \otimes \varphi_{i}\right) \otimes_{A}\left(1_{A} \# \bar{S}\left(t_{i(1)}\right) h\right) \\
=\sum_{i}\left(\left(t_{i(3)} \bar{S}\left(t_{i(2)}\right)\right) \cdot a \# t_{i(4)}\right) \otimes_{A}\left(1_{A} \otimes \varphi\right) \otimes_{A}\left(1_{A} \# \bar{S}\left(t_{i(1)}\right) h\right) \\
=\sum_{i}\left(a \# t_{i(2)}\right) \otimes_{A}\left(1_{A} \otimes \varphi\right) \otimes_{A}\left(1_{A} \# \bar{S}\left(t_{i(1)}\right) h\right) \\
=\sum_{i}\left(a \# h t_{i(2)}\right) \otimes_{A}\left(1_{A} \otimes \varphi\right) \otimes_{A}\left(1_{A} \# \bar{S}\left(t_{i(1)}\right)\right) \\
=\sum_{i}(a \# h)\left(1_{A} \# t_{i(2)}\right) \otimes_{A}\left(1_{A} \otimes \varphi_{i}\right) \otimes_{A}\left(1_{A} \# \bar{S}\left(t_{i(1)}\right) .\right.
\end{aligned}
$$

Obviously $N$ is left $A$-linear. Right $A$-linearity can be proved as follows:

$$
\begin{aligned}
& N((1 \# h \otimes \varphi) a)=N\left(h_{(1)} a \# h_{(2)} \otimes \varphi\right) \\
& \quad=\sum_{j} h_{(1)} \cdot a\left\langle\varphi_{j}, h_{(2)}\right\rangle\left\langle\varphi, t_{j}\right\rangle=N(1 \# h \otimes \varphi) a .
\end{aligned}
$$

(5) is satisfied since

$$
\begin{array}{r}
N\left(E^{1} \otimes_{A} U^{1}\right) E^{2}=1_{A} \bar{\nu}\left(e^{1} \otimes u^{1}\right)\left(1_{A} \# e^{2}\right) \\
\stackrel{(155)}{=} \quad 1_{A} \# \bar{\nu}\left(e^{1} \otimes u^{1}\right) e^{2}=1_{A} \# 1_{H} .
\end{array}
$$

Let us finally show that (6) holds. For all $a \in A$ and $\varphi \in I$, we have

$$
\begin{aligned}
E^{1} \otimes_{A} & U^{1} N\left(E^{2} \otimes_{A}(a \otimes \varphi)\right) \\
& =\sum_{i}\left(1_{A} \# t_{i(2)}\right) \otimes_{A}\left(1_{A} \otimes \varphi_{i}\right) N\left(a \# \bar{S}\left(t_{i(1)}\right) \otimes \varphi\right) \\
& =\sum_{i, j}\left(1_{A} \# t_{i(2)}\right) \otimes_{A}\left(a \otimes \varphi_{i}\right)\left\langle\varphi_{j}, \bar{S}\left(t_{i(1)}\right)\left\langle\varphi, t_{j}\right\rangle\right. \\
& \stackrel{(15)}{=} \quad\left(1_{A} \# 1_{H}\right) \otimes_{A}(a \otimes \varphi)
\end{aligned}
$$


Proposition 6.3. Let $H$ be a finitely generated projective Hopf algebra, and $A$ a left $H$-module algebra. The trace map $\operatorname{tr}: A \otimes J \rightarrow B=A^{H}$ is given by the formula

$$
\operatorname{tr}(a \otimes t)=t \cdot a .
$$

Proof. Observe that the map $g:(J \otimes A) \otimes_{A}(I \otimes A)$ in the Morita context associated to $I \otimes A$ is given by the formula

$$
g\left((t \otimes a) \otimes_{A}(\varphi \otimes b)\right)=\langle\varphi, t\rangle a b .
$$

Using the left handed version of (11), we compute, for $V=a \otimes t \in A \otimes J$ that

$$
\begin{gathered}
\operatorname{tr}(a \otimes v)=\chi\left(E^{1} g\left(U^{1} \chi\left(E^{2}\right) \otimes_{A} V\right)\right)=\sum_{i} \chi\left(\left(1_{A} \# t_{i}\right) g\left(\left(1_{A} \otimes \varphi\right) \otimes(a \otimes t)\right)\right) \\
=\sum_{i} \chi\left(\left(1_{A} \# t_{i}\right) a\langle\varphi, t\rangle\right)=\chi\left(\left(1_{A} \# t\right) a\right)=\chi\left(t_{(1)} \cdot a \# t_{(2)}\right)=t \cdot a .
\end{gathered}
$$

We can now apply Propositions 2.1, 2.2 and 3.2, and Theorem 4.5, and obtain the following result.

Corollary 6.4. Let $H$ be a finitely generated projective Hopf algebra, and $A$ a left $H$-module algebra. Assume that there exist $a_{i} \in A$ and $t_{i} \in \int_{l}^{H}$ such that $\sum_{i} t_{i} \cdot a_{i}=1$.

Then the following assertions are equivalent;

(1) $A$ is left $F B N$;

(2) $A \# H$ is left FBN and $A$ is left noetherian;

(3) $B$ is left $F B N$ and $A$ is left noetherian.

We recover [9, Theorem 2.3 and Corollary 2.4], [7, Theorem 8] and [10, Theorem 2.4]. If $H$ is Frobenius (e.g. if $k$ is a field, or $H=k G$ is a finite group algebra), then the space of left integrals is free. We can then take a free generator $t$ of $\int_{H}^{l}$ and the condition of the trace map means that there exists $a \in A$ such that $t \cdot a=1$. We observe that - in the case where the space of integrals is not free - the sufficient condition in Corollary 6.4 that there exist $a_{i} \in A$ and $t_{i} \in \int_{l}^{H}$ such that $\sum_{i} t_{i} \cdot a_{i}=1$ is weaker than the one given in [7, Theorem 8], where a single $t \in \int_{l}^{H}$ and $a \in A$ with $t \cdot a=1$ are needed.

In [9] and [10], it is stated that Corollary 6.4 holds under the weaker assumption (called (C1)) that $A$ is $A \# H$-quasi-projective. There seems to be a hole in the proofs in [9] and [10]: the proof of the implication 3$) \Longrightarrow 1$ ) uses the projectivity of $A$ as an $A \# H$-module (see Remark 4.6).

\section{Application to CORings}

Let $A$ be a ring. An $A$-coring is a coalgebra in the category of $A$-bimodules ${ }_{A} \mathcal{M}_{A}$. This means that we have two $A$-bimodule maps

$$
\Delta_{\mathcal{C}}: \mathcal{C} \rightarrow \mathcal{C} \otimes_{A} \mathcal{C} \text { and } \varepsilon_{\mathcal{C}}: \mathcal{C} \rightarrow A
$$

satisfying some coassociativity and counit axioms. The maps $\Delta_{\mathcal{C}}$ and $\varepsilon_{\mathcal{C}}$ are called the comultiplication and counit, and we use the Sweedler notation

$$
\Delta_{\mathcal{C}}(c)=c_{(1)} \otimes_{A} c_{(2)},
$$


where summation is understood implicitely. Corings were revived recently in [2], and we refer to [3] for a detailed discussion of all kinds of applications. The left dual $R={ }^{*} \mathcal{C}={ }_{A} \operatorname{Hom}(\mathcal{C}, A)$ is an $A$-ring, with multiplication rule

$$
(f \# g)(c)=g\left(c_{(1)} f\left(c_{(2)}\right)\right),
$$

for all $c \in \mathcal{C}$ and $f, g \in{ }^{*} \mathcal{C}$. The unit is $\varepsilon_{\mathcal{C}}$, and the ring morphism $i: A \rightarrow{ }^{*} \mathcal{C}$ is given by

$$
i(a)(c)=\varepsilon_{\mathcal{C}}(c) a .
$$

The $A$-bimodule structure on ${ }^{*} \mathcal{C}$ is then given by the formula

$$
(a f b)(c)=f(c a) b
$$

for all $a, b \in A, f \in \mathcal{C}^{*}$ and $c \in \mathcal{C}$.

$x \in \mathcal{C}$ is called grouplike if $\Delta_{\mathcal{C}}(x)=x \otimes_{R} x$ and $\varepsilon_{\mathcal{C}}(x)=1_{R} . \quad(\mathcal{C}, x)$ is then called an $R$-coring with a grouplike element. Now consider the map $\chi:{ }^{*} \mathcal{C} \rightarrow A$, $\chi(f)=f(x)$. It can be shown easily (see $[6])$ that $\left(\mathcal{C}^{*}, i, \chi\right)$ is an $A$-ring with a right grouplike character. We can also compute that

$$
B=A^{R}=\left\{a \in A \mid f(x a)=a f(x), \text { for all } f \in \mathcal{C}^{*}\right\} .
$$

Using the grouplike element $x$, we can define a right $\mathcal{C}$-coaction on $A$, namely

$$
\rho: \mathcal{C} \rightarrow A \otimes_{A} \mathcal{C} \cong \mathcal{C}, \rho(r)=1_{A} \otimes_{A} x a=x a .
$$

We can consider the subring of coinvariants

$$
A^{\mathrm{coC}}=\{a \in A \mid a x=x a\} .
$$

In general, $A^{\text {coC }}$ is a subring of $A^{R}$, and they are equal if $\mathcal{C}$ is finitely generated and projective as a right $A$-module.

An $A$-coring $\mathcal{C}$ is called Frobenius if there exist an $A$-bimodule map $\theta: \mathcal{C} \otimes_{A} \mathcal{C} \rightarrow A$ and $z \in C_{A}(\mathcal{C})$ (that is, $a z=z a$, for all $a \in A$ ) such that the following conditions hold, for all $c, d \in \mathcal{C}$ :

$$
\begin{gathered}
c_{(1)} \theta\left(c_{(2)} \otimes d\right)=\theta\left(c \otimes d_{(1)}\right) d_{(2)}, \\
\theta(z \otimes c)=\theta(c \otimes z)=1 .
\end{gathered}
$$

We refer to [5, Theorem 35] for the explanation of this definition. If $\mathcal{C}$ is Frobenius, $\mathcal{C}$ is finitely generated and projective as a (left or right) $A$-module, and ${ }^{*} \mathcal{C} / A$ is Frobenius (see [5, Theorem 36]). Then we also have (see [6, Sec. 3]) that

$$
Q=\left\{q \in{ }^{*} \mathcal{C} \mid c_{(1)} q\left(c_{(2)}\right)=q(c) x\right\} .
$$

It follows from [6, Theorem 2.7] or Theorem 3.1 that we have an isomorphism of $\left({ }^{*} \mathcal{C}, B\right)$-bimodules $\alpha: A \rightarrow Q$, given by

$$
\alpha(a)(c)=\theta\left(c a \otimes_{A} x\right),
$$

for all $a \in A$ and $c \in \mathcal{C}$. The inverse $\alpha^{-1}$ is given by $\alpha^{-1}(q)=q(z)$, and the left ${ }^{*} \mathcal{C}$-action on $A$ is

$$
f \cdot a=\theta\left(z_{(1)} f\left(z_{(2)}\right) \otimes_{A} x\right) .
$$

This can be verified directly as follows:

$$
\alpha\left(\alpha^{-1}(a)\right)=\theta\left(z a \otimes_{A} x\right)=\theta(a z \otimes x)=a \theta(z \otimes x)=a,
$$


and

$$
\begin{gathered}
\alpha\left(\alpha^{-1}(q)\right)(c)=\theta\left(c q(z) \otimes_{A} x\right)=\theta\left(c \otimes_{A} q(z) x\right)=\theta\left(c \otimes_{A} z_{(1)} q\left(z_{(2)}\right)\right. \\
=\theta\left(c \otimes_{A} z_{(1)}\right) q\left(z_{(2)}\right)=q\left(\theta\left(c \otimes_{A} z_{(1)}\right) z_{(2)}\right) \\
=q\left(c_{(1)} \theta\left(c_{(2)} \otimes z\right)\right)=q\left(c_{(1)} \varepsilon\left(c_{(2)}\right)\right)=q(c) .
\end{gathered}
$$

The trace map tr : $A \rightarrow B$ is given by

$$
\operatorname{tr}(a)=\theta\left(x a \otimes_{A} x\right) .
$$

Corollary 7.1. Let $(\mathcal{C}, x)$ be a Frobenius A-coring with a fixed grouplike element, and Frobenius system $(\theta, z)$, and assume that there exists $a \in A$ such that $\operatorname{tr}(a)=1$. Then the following assertions are equivalent.

(1) $A$ is right $F B N$;

(2) ${ }^{*} \mathcal{C}$ is right $F B N$ and $A$ is right noetherian;

(3) $B=A^{\text {coC }}$ is right $F B N$ and $A$ is right noetherian.

\section{Acknowledgement}

We thank Angel del Río and Sorin Dăscălescu for discussing with us the sufficiency of the quasi-projectivity assumption in the proof of 3$) \Longrightarrow 1$ ) in Theorem 4.5.

\section{REFERENCES}

[1] T. Albu and C. Năstăsescu, "Relative finiteness in module theory", Monographs Textbooks Pure Appl. Math. 84, Marcel Dekker, New York, 1984.

[2] T. Brzeziński, The structure of corings. Induction functors, Maschke-type theorem, and Frobenius and Galois properties, Algebr. Representat. Theory 5 (2002), 389-410.

[3] T. Brzeziński and R. Wisbauer, "Corings and comodules", London Math. Soc. Lect. Note Ser. 309, Cambridge University Press, Cambridge, 2003.

[4] S. Caenepeel, E. De Groot and G. Militaru, Frobenius functors of the second kind, Comm. Algebra 30 (2002), 5359-5391.

[5] S. Caenepeel, G. Militaru and Zhu Shenglin "Frobenius and separable functors for generalized module categories and nonlinear equations", Lect. Notes in Math. 1787, Springer Verlag, Berlin, 2002.

[6] S. Caenepeel, J. Vercruysse and Shuanhong Wang, Morita Theory for corings and cleft entwining structures, J. Algebra 276 (2004), 210-235.

[7] S. Dăscălescu, A. Kelarev, and B. Torrecillas: FBN Hopf module algebras, Comm. Algebra 25 (1997), 3521-3529.

[8] L. El Kaoutit, J. Gómez-Torrecillas and F. Lobillo, Semisimple corings, Algebra Coll. 11 (2004), 427-442.

[9] J. J. García and A. Del Río, Actions of groups on fully bounded Noetherian rings, Comm. Algebra 22 (1994), 1495-1505.

[10] T. Guédénon, Actions of Hopf algebras on fully bounded Noetherian rings, Beiträge Algebra Geom. 42 (2001), 395-400.

[11] L. Kadison, "New examples of Frobenius extensions", University Lect. Series 14, Amer. Math. Soc., Providence, 1999.

[12] C. Năstăsescu and S. Dăscălescu, Graded T-rings, Comm. Algebra 17 (1989), 3033-3042.

[13] B. Pareigis, When Hopf algebras are Frobenius Algebras, J. Algebra 18 (1971), 588-596.

[14] R. Wisbauer, On the category of comodules over corings, in "Mathematics and mathematics education (Bethlehem, 2000)", World Sci. Publishing, River Edge, NJ, 2002, 325-336.

Faculty of Engineering, Vrije Universiteit Brussel, VUB, B-1050 Brussels, Belgium

E-mail address: scaenepe@vub.ac.be

$U R L:$ http://homepages.vub.ac.be/〜scaenepe/

Faculty of Engineering, Vrije Universiteit Brussel, VuB, B-1050 Brussels, Belgium

E-mail address: tguedeno@vub.ac.be, guedenon@caramail.com 